\title{
Characterization of the cooling channels of First Wall Mock-up dedicated to the HCPB-TBM qualification
}

\author{
A. Abou-Sena, L.V. Boccaccini, B.E. Ghidersa, K. Zinn \\ Karlsruhe Institute of Technology (KIT), 76344 Eggenstein-Leopoldshafen, Germany
}

\begin{abstract}
The First Wall (FW) of the EU Helium Cooled Pebble Bed (HCPB) Test Blanket Module (TBM) faces the fusion plasma and experiences high heat fluxes; therefore its cooling channels design is a key R\&D task for qualifying the HCPB TBM for the fusion reactors ITER and DEMO. Within the manufacturing and qualification activities performed in KIT for the HCPB TBM, a First Wall Mock-up (FWM) was designed and manufactured. The objective of this study is to characterize the hydrodynamic behaviour of the FWM cooling channels by investigating the coolant pressure loss for each channel. The FWM has a shape of rectangular prism $(710 \mathrm{~mm} \times 405 \mathrm{~mm} \times 45 \mathrm{~mm})$ and $10 \mathrm{U}$-shaped cooling channels which have a square cross section $(15 \mathrm{~mm} \times 15 \mathrm{~mm})$ with rounded corners of $4 \mathrm{~mm}$ radius. The FWM was integrated into an experimental gas loop that has the relevant instrumentation and piping system for measuring and controlling the gas (e.g. compressed air of 6 bar abs.) flow parameters. This paper presents the experimental results of measuring the pressure loss across the FWM cooling channels and discusses its main causes. In addition, the experimental results are compared with theoretical values obtained from relevant models and formula available in the literature. The results of this study will support the qualification of the HCPB TBM mock-ups which will be tested at fusion-relevant heat flux and helium cooling in the Helium Loop Karlsruhe (HELOKA) facility.
\end{abstract}

